\title{
Percutaneous First Metatarsocuneiform Joint Arthrodesis: Treatment of Severe Recurrent Forefoot Deformity Complicated by an Infected Wound
}

\author{
by Sergey Berezhnoy, $\mathrm{MD}, \mathrm{PhD}^{1} \otimes$
}

The Foot and Ankle Online Journal 5 (3): 2

Percutaneous surgery for some forefoot deformities is now accepted by some orthopaedic surgeons as a viable fixation technique in the presence of infection. In most cases percutaneous surgery refers to distal procedures limiting its application in hallux valgus surgery. The purpose of this report was to develop a percutaneous first metatarsocuneiform joint arthrodesis technique in the presence of infection. This case report represents an example of successful surgical treatment of a severe recurrent forefoot deformity complicated by an infected wound. The arthrodesis fused three months after surgery. The effectiveness and reproducibility of a percutaneous modification in the Lapidus procedure should be investigated further.

Key words: Percutaneous foot surgery, first metatarsocuneiform joint arthrodesis, minimally invasive technique, Lapidus procedure.

\section{Accepted: February, $2012 \quad$ Published: March, 2012}

$\mathrm{P}$ ercutaneous surgery to correct forefoot deformities is now accepted to a greater or lesser extent by many orthopaedic surgeons. ${ }^{1}$ There are few published reports about minimal invasive first metatarsal basal osteotomies. ${ }^{2}$ There does not appear to be any recent publications of percutaneous first metatarsocuneiform joint ( $1^{\text {st }}$ MTCJ) arthrodesis in the presence of infection. A case report is presented of a $1^{\text {st }}$ MTCJ arthrodesis using percutaneous screws in the presence of an infected wound.

Address correspondence to: S. Berezhnoy, MD, PhD, 125284, 2nd Botkinskiy proezd, 5/5, Moscow, Russia. Email: Doktor@berezhnoi.ru.

${ }^{1}$ Medincentre of the Main Administration for Service to the Diplomatic Corps by the Ministry of Foreign Affairs of Russia. Orthopaedic surgeon. 125284, 2nd Botkinskiy proezd, 5/5, Moscow, Russia.

\section{Case report}

A 73 year-old female underwent hallux valgus surgery 15 years ago. No documents were uncovered concerning the surgical technique or circumstances of surgery. For the last 5 years the patient has noticed a gradual recurrence of the forefoot deformity. One month prior to presentation, she developed acute first metatarsophalangeal ( $1^{\text {st }}$ MTP) joint inflammation with fever up to 39 degrees Celsius. A draining, purulent wound appeared a few days later. The patient was admitted to the septic department of another hospital. She was treated conservatively for several days with some improvement and then transferred to our clinic. 

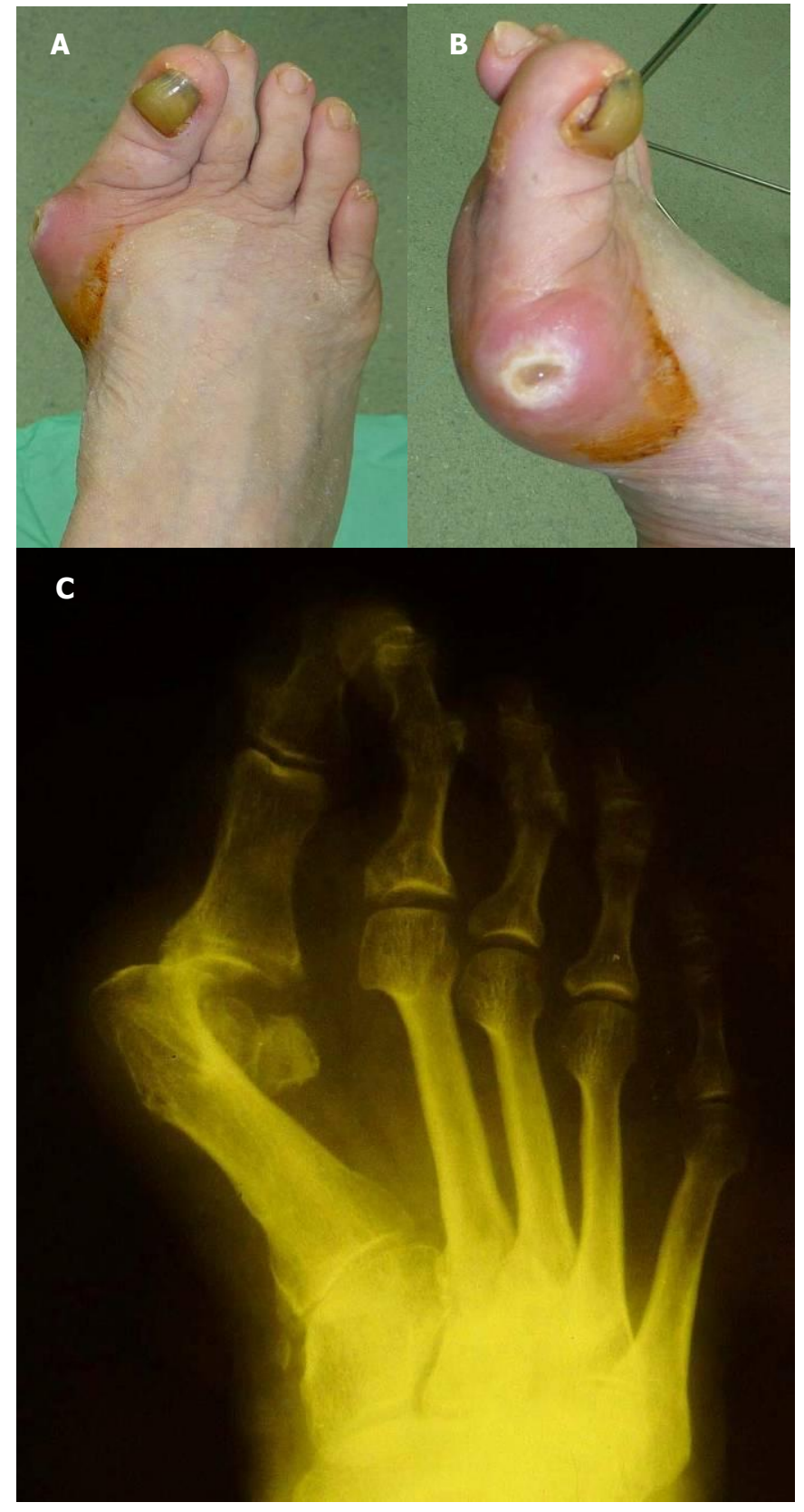

Figure 1A, 1B and 1C Photos ( $A$ and $B$ ) and anteroposterior radiograph (C) of the foot on admission.

Clinical evaluation on admission revealed a patient that was partial weightbearing with crutches. She presented with severe right foot hallux abductovalgus deformity, a thin postoperative scar about $11 \mathrm{~cm}$ on the dorsomedial aspect of the foot and a purulent wound $1.2 \mathrm{~cm}$ on the medial aspect of the $1 \mathrm{st}$ MTP joint.
There was moderate edema and erythema around the wound. (Figs. 1A and 1B) Body temperature was $37.3^{\circ} \mathrm{C}$. A non-weightbearing right foot radiograph revealed a first intermetatarsal angle 30 degrees, hallux valgus angle 55 degrees and almost complete big toe lateral dislocation. There were no signs of bone destruction or osteomyelitis. (Figure 1C). Also there were no clinical or radiological signs of adjacent joint involvement (1st interphalangeal joint and 1st MTCJ) associated with the inflammatory process.

A diagnosis was made including severe recurrent right foot hallux abductovalgus deformity with lateral dislocation of the hallux. This was complicated by a draining, purulent wound and 1st MTP joint purulent arthritis. The patient underwent surgical debridement 1 day after admission including a partial synovectomy and primary wound closure. Percutaneous 1st MTCJ arthrodesis with two compression screws fixation was performed. This included a percutaneous non fixed Akin osteotomy. Written informed consent was obtained from the patient for publication of this case report and accompanying images.

\section{Surgical technique}

Initially, the wound was debrided by wide excision of its edges. A partial 1st MTP joint synovectomy and 1st metatarsal head resection was performed. The wound was covered with a sterile gauze. Two - $2 \mathrm{~mm}$ Kirschner wires were inserted percutaneously on the dorsomedial aspect of the foot; one in the base of the first metatarsal and the other in the medial cuneiform. A Hinterman retractor was used to expose the 1st MTCJ. The 1st MTCJ was then opened under fluoroscopy and a Beaver 64 blade was used to make a $0.5 \mathrm{~cm}$ longitudinal incision between the 2 Kirschner wires. Complete removal of the MTCJ cartilage was performed using small burrs, periosteal elevators, curette and suction. Several perforations and notches were created to the subchondral bone with a Shannon burr tip and cutting edge. Bone debris was left to facilitate fusion. The retractor and Kirschner wires were then removed. 


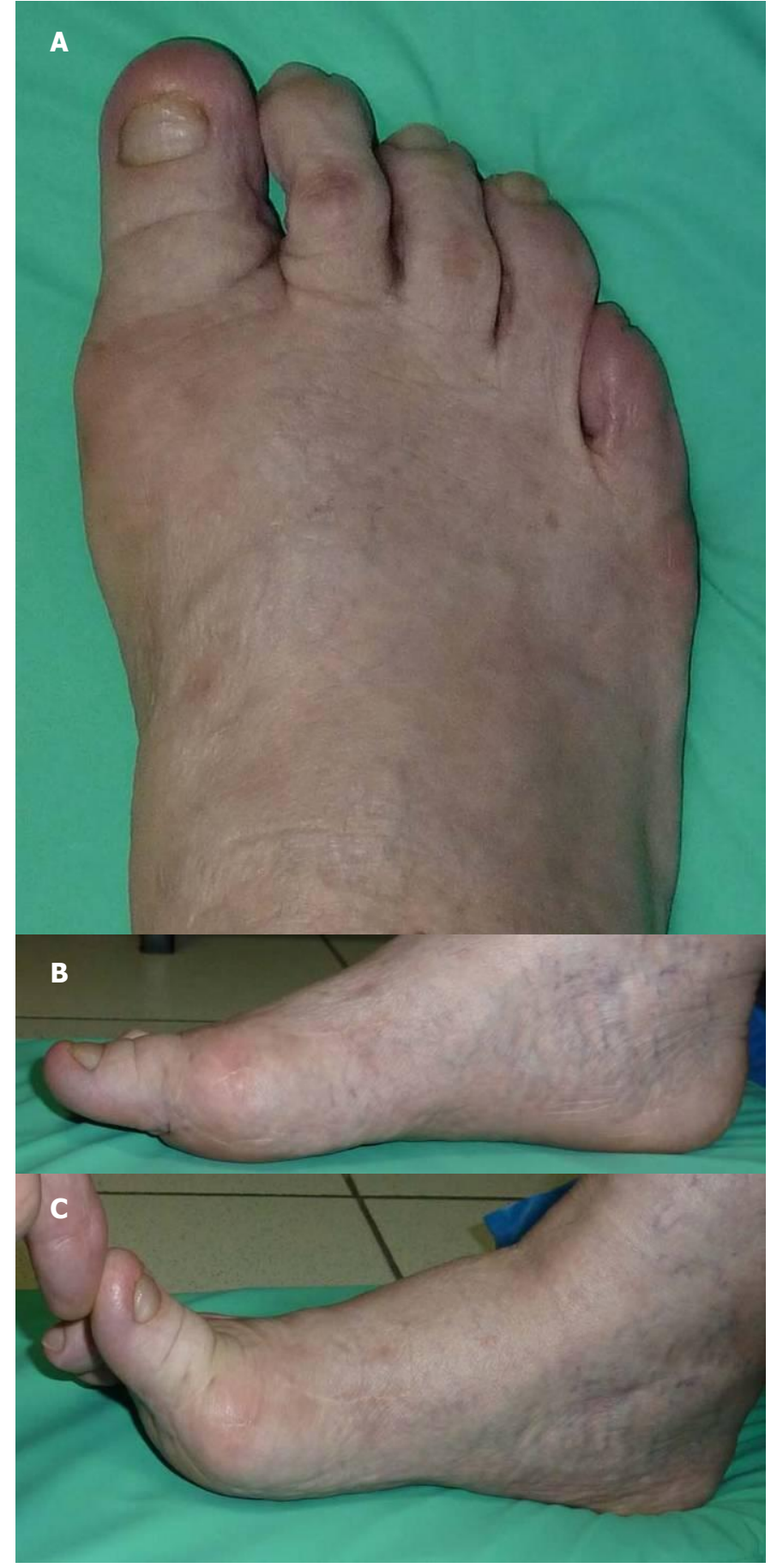

Figure 2A, 2B and 2C Photos (A, B and C) of the foot at 9 months follow up showing excellent big toe alignment and 1st MTP joint good range of motion.

The first intermetatarsal angle was reduced using a clamp, placed through a wound at the level of the first metatarsal head and a skin puncture made laterally at the level of the second metatarsal neck.
The desired position of the first metatarsal was then fixed with two $4 \mathrm{~mm}$ partially threaded cannulated screws. 2- $1 \mathrm{~mm}$ Kirschner wires were used as the guides for screw introduction. The first screw was placed from the dorsomedial aspect of the medial cuneiform into the plantar aspect of the first metatarsal. The second screw was placed from the dorsomedial surface of the first metatarsal base to the intermediate cuneiform. A percutaneous, non-fixed Akin osteotomy procedure was performed and 3 small midfoot wounds were closed. The wound was completely closed without a drain. Elastic dressing was applied with a big toe fixed in slight hypercorrection.

\section{Postoperative treatment}

The patient was placed in a postoperative shoe. Once day following surgery, the patient was instructed to ambulate with crutches as tolerated. Her medications included the use of Meronem $2 \mathrm{~g}$ and Xefocam $16 \mathrm{mg}$ daily for five days to address symptomatic treatment. The patient was discharged from the hospital on the sixth day. Stitches were removed on the twelfth day. All the wounds healed uneventfully. The patient was kept partial weightbearing with crutches for 3 weeks postoperatively.

At 9 months, clinical evaluation revealed excellent big toe alignment with the 1st MTP joint good range of motion (Figs. 2A, B and C). The patient could ambulate in a normal shoe without restrictions. Computerized tomography and standard weightbearing radiographic studies showed 1st MTCJ fusion, good first ray alignment with 1st intermetatarsal angle 19 degrees and hallux valgus angle 13 degrees. (Figs. 3A, B and C).

\section{Discussion}

Reconstruction of the forefoot deformity recurrences is always a challenge for the orthopaedic surgeon. A recurrent deformity in the presence of an infected wound made this case much more complex. In order to decrease the risk of osteomyelitis and possibly contaminate an infected site with the proposed 


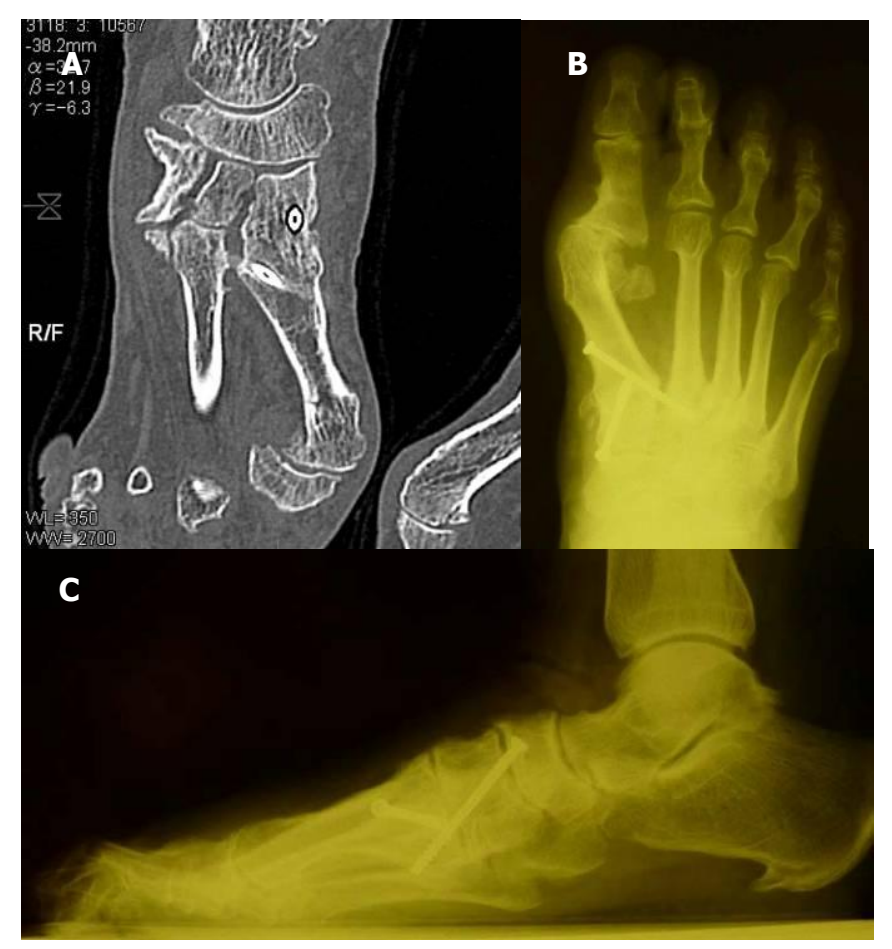

Figure 3A, 3B and 3C Computed Tomography at nine months follow up showing $1^{\text {st }}$ metatarsocuneiform joint fusion (A); anteroposterior (B) and lateral (C) weightbearing radiographs of the foot showing good first ray alignment.

Lapidus fusion, we proposed a percutaneous MTCJ fusion. This approach provided a safe and relatively simple solution in reducing the first intermetatarsal angle an ensure hallux reduction and wound healing. In combination with a percutaneous Akin procedure, we managed to achieve excellent first ray realignment. 3,10,11 The use of cannulated screws facilitated the fixation process. ${ }^{6,8}$ Early weightbearing after the Lapidus procedure is now a viable choice after bunion correction. ${ }^{4,9,12} \quad$ Immediate postoperative weightbearing protocol varies among surgeons, institutions and geographic regions. ${ }^{5}$ Since 2008, routine weightbearing protocol in our department includes immediate ambulation in a postoperative shoe with the use of one crutch for three weeks. Taking into account the age of our patient, we recommended the use of two crutches. The arthrodesis fused by 3 months after surgery.

(C) The Foot and Ankle Online Journal, 2012
Placement of the screw into the medial cuneiform was technically the most demanding step of the procedure because of the low angle between the skin and bone surfaces and the directional placement of the screw. To solve this problem one should make skin incision 1-1.5 $\mathrm{cm}$ proximal to the naviculocuneiform joint line and place the screw entry point at the dorsal margin of the medial cuneiform articular surface. To avoid soft tissue irritation over the heads of the screws it is better to use compressive headless cannulated screws for complete intraosseous fixation.

The case of the successful treatment of a severe recurrent forefoot deformity complicated by an infected wound is presented. A minimally invasive approach proposed by the author has shown itself to be safe and technically not very demanding. However, the surgeon must be familiar with percutaneous foot surgery techniques. The effectiveness and reproducibility of the Lapidus procedure using a percutaneous technique should be demonstrated by further investigations.

\section{Conflict of interest statement}

The author declares that there are no conflicts of interest.

\section{References}

1. Bauer T, de Lavigne C, Biau D, de Prado M, Isham S, Laffenêtre $O$. Percutaneous hallux valgus surgery: a prospective multicenter study of 189 cases. Orthop Clin N Am 2009 40: 505514. PubMed]

2. De Lavigne C, Rasmont Q, Bao Hoang. Percutaneous double metatarsal osteotomy for correction of severe hallux valgus deformity. Acta Orthop Belg 2011 77: 516-521.[PubMed]

3. Barouk LS, Barouk P, Baudet B, Toullec E. The great toe proximal phalanx osteotomy: the final step of the bunionectomy. Foot Ankle Clin 2005 10: 141-155. [PubMed]

4. Blitz N. Why the Lapidus bunionectomy is the best procedure for severe bunions. Podiatry Today 2011 24: 44-49. Website]

5. Blitz, DeHeer P. Early weightbearing after Lapidus: is it possible? Podiatry Today 2004 17: 46-54. [Website]

6. Brown G, McCarthy $\mathrm{T}$, Bourgeault C, Callahan D. Mechanical performance of standard and cannulated $4.0-\mathrm{mm}$ cancellous bone screws. J Ortho Res 2000 18(2): 307-312. [PubMed] 
7. Coetzee JC, Wickum D. The Lapidus procedure: a prospective cohort outcome study. Foot Ankle Int. 2004 25: 526-531. [PubMed]

8. Cohen D, Parks B. Screw fixation compared to H-locking plate fixation for first metatarsocuneiform arthrodesis: a biomechanical study. Foot Ankle Int 2005 26: 984-989. [PubMed]

9. Basile P, Cook EA, Cook JJ. Immediate weight bearing following modified Lapidus arthrodesis. J Foot Ankle Surg 2010 49: 459-64. PubMed

10. De Prado M, Ripoll PL, Golano P. Cirugia percutanea del pie. Masson 2003: 7-14. Website]

11. Gérard M., Stern R., Assal M. The modified Lapidus procedure. Orthopedics 2008 31: 230-234. [PubMed]

12. Kazzaz S., Singh D. Postoperative cast necessity after a Lapidus arthrodesis. Foot Ankle Int 2009 30: 746-751. [PubMed]

13. Lapidus PW. A quarter of a century of experience with the operative correction of the metatarsus varus primus in hallux valgus. Bull Hosp Joint Dis 1956 17: 404-421. [PubMed]

14. O'Kane C, Kilmartin E. The rotation Scarf and Akin osteotomy for the correction of severe hallux valgus. The Foot 2002 12: 203-212. [Website]

15. Thompson IM, Bohay DR, Anderson JG. Fusion rate of first tarsometatarsal arthrodesis in the modified Lapidus procedure and flatfoot reconstruction. Foot Ankle Int 2005 26: 698-703. [PubMed] 